\title{
El parque ecoarqueológico Xcaret: apuntes sobre su modelo de negocio y organización
}

\author{
Martín Manuel Checa-Artasu* \\ Universidad Autónoma Metropolitana \\ Unidad Iztapalapa
}

\section{Resumen}

En este trabajo se analiza una serie de aspectos en torno al parque ecoarqueológico Xcaret, en la Riviera Maya, en Quintana Roo, y se hace hincapié en el componente organizacional y empresarial que lo sustenta, por ser un modelo de negocio a considerar en el marco del actual desarrollo turístico del estado. Con casi tres décadas de operación, el grupo empresarial que gestiona Xcaret es líder en servicios turísticos en México. Ese liderazgo está relacionado con una gestión empresarial que trata de cerrar el ciclo de procesos derivados del servicio turístico que oferta y la articulación de forma eficaz de un discurso donde la sostenibilidad se convierte en el eje vertebrador de la empresa en todas sus facetas.

\section{PALAbras Clave}

Xcaret, gestión empresarial, responsabilidad social, turismo, Quintana Roo. 


\title{
The eco-archeological park Xcaret, some notes on its business and organizational models
}

\author{
Martín Manuel Checa-Artasu \\ Universidad Autónoma Metropolitana \\ Unidad Iztapalapa
}

\begin{abstract}
This article analyzes various aspects of the eco-archeological park Xcaret in the Mayan Riviera in Quintana Roo, Mexico and it focuses on its organizational and entrepreneurial components, considering it as a model to be followed throughout the state. After almost three decades of operation the corporate group managing Xcaret is a leading provider of tourist services in Mexico. This leadership is linked to the entrepreneurial management that has tried to close the process cycles of the tourist supply and the articulation of an efficient way of incorporating sustainability as an axis in the fundamental structure of the enterprise.
\end{abstract}

\section{KEY WORDS}

Xcaret, entrepreneurial managements, social responsibility, tourism, Quintana Roo 


\section{Introducción}

Este trabajo, eminentemente descriptivo, es un análisis de algunas cuestiones relativas al modelo de negocio y organización que se ha gestado en torno al funcionamiento del parque ecoarqueológico Xcaret, situado en la Riviera Maya, en el estado de Quintana Roo. Este análisis es la continuación de anteriores trabajos que examinaban específicamente las características de este parque de ocio como lugar turístico y su relación con el patrimonio cultural allí existente, que sirvieron de excusa para su formalización (Checa-Artasu, 2008 y 2009). Quiero hacer hincapié en el componente organizacional y empresarial que lo sustenta por ser un modelo de negocio a considerar en el marco del actual desarrollo turístico quintanarroense. El parque ecoarqueológico Xcaret, gestionado por el consorcio turístico del mismo nombre, es un caso paradigmático en muchos sentidos, ya que se presenta como la readaptación tanto de espacios naturales como de elementos patrimoniales para una oferta turística que combina ambos aspectos con actividades lúdicas coincidentes con las estrategias del turismo de sol y playa que se dan en la Riviera Maya.

Tras casi tres décadas en ejercicio el grupo empresarial que gestiona Xcaret (Grupo Xcaret) es una empresa líder en servicios turísticos en México, que, de forma descentralizada, dirige otros establecimientos de similares características en el Caribe mexicano, como el parque ecoturístico Xel-Há y el parque Xplor. Además, tiene vínculos accionariales con otros grupos turísticos que operan en la zona y que le permiten recibir sus sinergias. Todo ello manteniendo un posicionamiento ideológico que promueve la sostenibilidad y el respeto al medio ambiente como motor de su desarrollo y que vertebra ese posicionamiento hacia el ejercicio de responsabilidad social en sus distintos ámbitos de acción.

\section{El parque Xcaret como producto turístico: volumen de visitantes}

El parque ecoarqueológico Xcaret está situado en el municipio de Solidaridad, en la parte central de la Riviera Maya. Su proximidad relativa al gran polo turístico de Cancún, a unos 60 kilómetros, siguiendo la carretera federal núm. 180 Cancún-Chetumal, y su cercanía con Playa del Carmen, a cinco kilómetros —una población en fase expansiva de 100383 habitantes y 23428 habitaciones en uso 
en 2005, con gran capacidad de atracción turística dados los servicios que reúne (INEGI, 2005; Campos Cámara, 2007: 89)—, le garantizan un flujo de visitantes, ya sea de forma individualizada o a través de turoperadores, agencias o cruceros, y lo convierten en un lugar enormemente referenciado y bien posicionado dentro de la oferta de productos turísticos de la zona. Por ejemplo, de 2005 a 2010 registró un promedio de poco más de un millón de visitantes al año, cifra que, comparada con el flujo promedio anual de turistas a Quintana Roo en el mismo periodo, señala que $15.16 \%$ de los visitantes al estado pasaron por el parque (véase cuadro 1).

CUADRO 1. Comparativa entre número de visitantes al parque Xcaret y turistas totales a Quintana Roo (2005-2010)

\begin{tabular}{lccccccc} 
& 2005 & 2006 & 2007 & 2008 & 2009 & 2010 & $\begin{array}{l}\text { Promedio } \\
\text { 2005-2010 }\end{array}$ \\
& 1340000 & 880936 & 1065993 & 1073471 & 872913 & 1053606 & 1047819 \\
$\begin{array}{l}\text { Visitantes a Xcaret } \\
\begin{array}{l}\text { Turista totales a } \\
\text { Quintana Roo }\end{array}\end{array}$ & 6112670 & 5935316 & 7005387 & 8025745 & 6855385 & 7518458 & 6908826 \\
\hline $\begin{array}{l}\text { Promedio visitantes } \\
\begin{array}{l}\text { Xcaret/turistas } \\
\text { totales Quintana Roo }\end{array}\end{array}$ & 21922 & 14842 & 15217 & 13375 & 12733 & 14014 & 15166 \\
\hline
\end{tabular}

Fuente: Elaboración propia a partir de Grupo Xcaret, 2008 y 2009, y con datos de Promotora Xcaret e indicadores turísticos de la Secretaría de Turismo del estado de Quintana Roo.

Un análisis más detallado de quienes arribaron a Xcaret entre 2005 y 2010 nos hace intuir una relativa estabilidad en cuanto al volumen de personas que recibe el parque y probablemente marca su techo de ocupación. De igual forma, en el mismo periodo se apunta un descenso en el promedio de visitantes a Xcaret respecto al total de turistas a Quintana Roo debido a la aparición de espacios similares u otros de características diferentes, competidores de esta oferta. Así, mientras en 2004 recibía $37.5 \%$ del total de visitantes a esa entidad (Ravelo y Estolano, 2004), en 2010 su flujo era de $14 \%$ del total de turistas en el estado. El mismo análisis permite deducir algunos comportamientos de los visitantes y de la empresa. La gráfica 1 muestra la distribución de los flujos de asistentes al parque a lo largo de un mes (marzo de 2006). La mayor concurrencia se observa en los días centrales de las semanas del mes analizado, pues muchos paquetes turísticos que se ofertan 
para conocer la Riviera Maya, ya sean los vendidos en Estados Unidos y Canadá o los ofertados desde Europa, tienen una duración de siete a nueve días. Los días centrales de esos periodos son los que se destinan a ir al parque, y esa asistencia baja los primeros y los últimos días de la semana, destinados a la llegada y la salida de México. Ese hecho explica la agresividad en la mercadotecnia y promoción de la empresa, evidenciada en la notable presencia publicitaria de Xcaret en hoteles, agencias de viajes, turoperadores y otros establecimientos turísticos de Cancún y la Riviera Maya, e incluso en posiciones tan alejadas pero estratégicas como los puntos informativos ubicados en el aeropuerto internacional Benito Juárez, en la ciudad de México, y en el de Guadalajara.

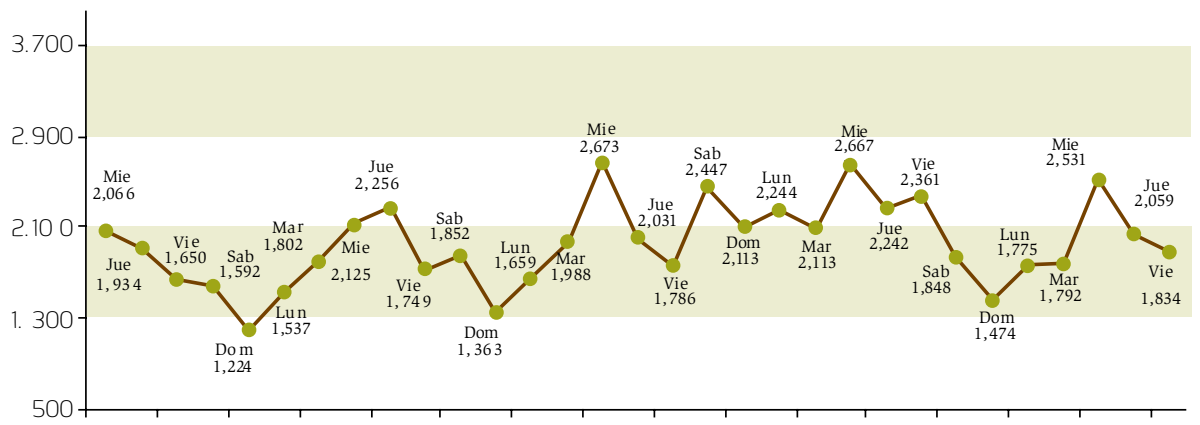

GRÁFICA 1. Distribución de los visitantes por día (marzo de 2006)

\section{El parque Xcaret: medio natural y morfología}

Xcaret integra diversas actividades recreativas aprovechando los recursos naturales con los que cuenta: 60 hectáreas de selva baja combinada con manglares junto a una pequeña caleta en medio de un kilómetro de litoral rocoso y coralífero; pero también utiliza algunos recursos pseudonaturales construidos para el proyecto edilicio de Xcaret.

El parque se inauguró en 1992 con un espacio inicial de 40 hectáreas, que se ha ampliado en el transcurso de los años. Previamente, en 1988, había obtenido una concesión por 15 años de 13345 metros cuadrados para el uso y aprovechamiento de las instalaciones construidas en terrenos de una zona federal marítimo-terrestre (Meraz, 2008), relativos al litoral referido, que colindaban con los terrenos adquiridos a privados a partir de 1986 y que conforman el parque en la actualidad. 
La construcción supuso una importante transformación del entorno prístino. El río, de un kilómetro, que discurre por el interior del parque y sirve de marco a diversas actividades acuáticas lúdicas, originalmente eran dos riachuelos de apenas 500 metros, unidos entre sí por grietas, cenotes y cavernas. Este sistema hidrogeológico fue conectado, ampliado y abierto al aire libre, para lo cual se realizaron voladuras de algunas cavernas en su construcción inicial, con objeto de proveer un mejor uso y disfrute a los visitantes. De igual forma, los recorridos subterráneos que tiene el parque se hicieron aprovechando unas cavernas presentes en la zona, pero creando unas cavidades artificiales, que conforman una red de cuatro kilómetros de túneles. También se desarrolló una estrategia de crecimiento del mínimo arrecife coralino existente, y se dotó al parque de un arrecife artificial que hoy, a manera de acuario, sirve de atracción turística y ha devenido referente internacional en la regeneración de entornos coralíferos (Sánchez, Jordán y Raigoza, 2000). Más tarde se levantó un pequeño cerro artificial donde se ubicó un camposanto y una capilla de un típico e idealizado pueblo mexicano. Este lugar es usado para que los asistentes disfruten las tradiciones festivas, como el Día de Muertos, o es rentado para bodas a gente adinerada o famosa. Curiosamente, el cerro se ha convertido en un nuevo accidente geográfico en una costa libre de relieves elevados, por lo que sirve como punto de referencia para la navegación deportiva. Conviene decir que todas esas modificaciones fueron el fruto de un proceso promovido y deseado por el fundador del parque, Miguel Quintana Pali, y transmitido a la organización empresarial, la cual derivó inversiones hacia ese resultado. Xcaret es, por tanto, la recreación de una realidad deseada, justificada proyectualmente (Rodríguez, 2000: 3; Howard, 2002; Lira, 2002).

\section{El parque Xcaret: interrelación entre patrimonio y cultura}

En el parque se encuentra la zona arqueológica de Xcaret, con secuencias cronológicas del Clásico Tardío y del Posclásico Temprano y Tardío (600-900/1000 d. C., 900/1000-1200 d. C. y 1200-1550 d. C.). El yacimiento se divide en siete estructuras, de las cuales cinco quedan dentro del parque de ocio, que corresponden a los restos de la antigua ciudad de P'ol'e. Además, se incorporan los restos de una capilla de ramada del siglo XVI, similar a las utilizadas por frailes itinerantes (Wyllys Andrews y Andrews, 1975: 11). 
La excavación arqueológica de gran parte de las estructuras, así como su consolidación, se inició en 1986 por el Instituto Nacional de Antropología e Historia (INAH) y coincidió con la compra de los terrenos del futuro parque, cuando se proyectaban las obras en él. Esta circunstancia derivó en una larga controversia entre el INAH y la Promotora Xcaret por el uso del patrimonio y la situación de éste una vez construido el parque. Ambos acordaron un convenio de colaboración mediante el cual la Promotora Xcaret da anualmente 774000 pesos al INAH por el uso de los restos arqueológicos dentro del parque (Rodríguez, 2000). Las cinco estructuras, que han sido consolidadas y limpiadas para su observación, cuentan con la señalética propia del INAH, conformada por unos croquis y unos paneles explicativos que atienden más a la estructura arqueológica en sí misma, minimizando el contexto donde ésta se encuentra y la interpretación del marco cronológico donde ubicar los restos.

Por su parte, para la empresa gestora del parque, la presencia de los restos arqueológicos es el acicate para su utilización simplista (Razo Ruiz, 2004: 46). Sólo es el vertebrador de algunas rutas guiadas que explican el pasado histórico de la zona que se combinan con otras actividades ligadas a los recursos pseudonaturales creados en el parque (mariposario, delfinario, esnórquel, buceo, etc.). Se trata de productos preparados para un perfil de público que acude al lugar no tanto para conocer el patrimonio arqueológico, sino para divertirse con las atracciones naturales y pseudonaturales que el parque ofrece (Cortés de Brasdefer, 2005: 257). Estos restos patrimoniales, además, fueron la excusa para que los gestores de Xcaret optaran por una recreación espectacularizante de la cultura maya, ya en forma de una supuesta ceremonia celebrada en el pueblo maya de cartón piedra erigido en el parque, ya con una representación de un juego de pelota que no corresponde con ningún resto arqueológico del yacimiento, pero que es réplica, construido in situ, de los de Copán o Monte Albán. El visitante asume pues una visión de cómo era una población maya del Posclásico Medio según los diseñadores del parque, no según la ciencia arqueológica (Mateos Vega, 2007). Paradójicamente, los dueños, al querer ampliar la oferta de actividades ligadas a la presencia de lo maya, han buscado la reproducción fiel de ese pasado, e incluso la justificación científica de sus recreaciones. Así, en abril de 2008, la Promotora Xcaret firmó un convenio de colaboración con el Centro de Estudios Mayas de la Universidad Nacional Autónoma de México (UNAM), que indica la asesoría especializada, así como su colaboración para temas culturales muy diversos, por ejemplo festivales, exhibiciones, espectáculos y otros productos culturales que se realizan en el lugar. 
Con el paso del tiempo, el discurso de "lo maya" al que se adscribía Xcaret se volvió uno que daba mayor valor a una supuesta idea de "lo mexicano". De ese modo, a las ruinas del yacimiento maya y a las reconstrucciones de esa cultura se añadieron una capilla y un camposanto de un típico y periclitado pueblito mexicano, una impecable mina de plata y una perfecta hacienda henequenera -estructura socioeconómica del siglo XIX en la Península de Yucatán- donde se ubicó un museo de arte popular mexicano. También se han construido un mariposario, un aviario, una desfibradora de henequén y una destilería tequilera. Asimismo, se han incorporado elementos de la mexicanidad, como las representaciones in situ de los rituales de los voladores de Papantla, originarios del estado de Veracruz. Esta idea de la mexicanidad culmina con una doble representación diaria del espectáculo Xcaret México Espectacular en una inmensa palapa, construcción propia de esta zona, reconvertida en un tradicional palenque de espectáculos mexicano donde abundan los trajes y las danzas tradicionales de todo el país, ejecutados por cerca de 300 artistas que dan un sentido folclórico, edulcorado y turístico de la identidad mexicana.

\section{Emprendedores en el proyecto empresarial de Xcaret}

Xcaret se presenta como un proyecto pretendido largo tiempo por su creador, Miguel Quintana Pali, y que esconde la pertinaz insistencia del emprendedor convencido del modelo de negocio que busca desarrollar: vincular el ocio con la sustentabilidad del medio natural.

Nacido en Boston en 1946, debido quizás a los quehaceres de su padre, Carlos Quintana (1912-1987), relacionados con la Comisión Económica para América Latina y el Caribe (CEPAL), de la que llegaría a ser secretario ejecutivo de 1967 a 1972, se cría en la ciudad de México, donde estudia Ingeniería Química en el Instituto Tecnológico y de Estudios Superiores de Monterrey y cursa cuatro años de la Licenciatura en Arquitectura de la Universidad Iberoamericana.

La actividad empresarial de Quintana Pali comienza en 1979 con la comercialización puerta a puerta de una lámpara de su propia creación. Más tarde vende muebles y objetos de decoración en una tienda ubicada en el centro comercial Plaza Satélite, en el Estado de México, y seis años después funda la marca Pali y una pequeña red de tiendas de artículos de decoración (Howard, 2002). Esa iniciativa y la búsqueda de posibles clientes lo ponen en contacto con algunos de los operadores que construían 
Cancún a principios de la década de los ochenta y que son clave para entender la gestación de Xcaret como parque de ocio entre 1986 y 1992. Hay que recordar que en ese entonces Cancún empezaba a ser vista como una zona de enorme potencial para el desarrollo de negocios turísticos (Martí, 1985).

En 1986, Quintana Pali compra cinco hectáreas en una zona de selva con cenotes y manglares conocida como Rancho Xcaret. La idea inicial era construir una casa en las cercanías de uno de los cenotes (Rodríguez, 2000). Tras ese primer intento, proyecta una urbanización de 30 villas a manera de resort, y luego muda el proyecto al diseño de un parque de ocio. Esos dos sucesos y la necesidad de inversores lo hacen acercarse a diversos agentes que desde hacía una década operaban en Cancún y que en esos años comenzaban un proceso de expansión hacia el sur, es decir, desde Cancún hasta Tulum (Córdova Ordoñez y García Fuentes, 2003: 122).

Entre ellos destacan el arquitecto Román Rivera Torres y los hermanos Óscar, Marcos y Carlos Constandse Madrazo, los dos primeros ingenieros y el tercero arquitecto, todos copropietarios de las hectáreas que Quintana Pali había comprado y, a la vez, socios de la firma constructora Ritco y Asociados, que años atrás había participado en la construcción del polo turístico de Cancún. Esta empresa estaba integrada, aparte de los citados, por el economista y contador público Francisco Córdova Lira. Ritco se funda en 1974 y a la fecha ha realizado todo tipo de obra pública: vivienda, locales comerciales y, especialmente, diversos complejos turísticos en Cancún y en la Riviera Maya. Hoy en día se posiciona como un grupo inmobiliario compuesto por ocho empresas que tienen como principales accionistas a los miembros de la familia Constandse Madrazo.

En 1990, Quintana Pali presenta su proyecto de parque lúdico a esos agentes para comprar cerca de 60 hectáreas que les pertenecían, amén de tratar de captarlos como posibles inversores. Esta transacción permitió constituir y delimitar los socios de Promotora Xcaret, gestora del proyecto del parque. Entonces, a ella se asociaron Quintana Pali, los hermanos Constandse (quienes tendrían $30 \%$ de la firma y para ello crearían el Grupo Alquimia), y Rivera Torres y Córdova Lira (quienes quedaron al margen del accionariado de la nueva empresa). Este último, tiempo después, se vincularía al proyecto como director ejecutivo y en 2003 sería socio accionarial de Xcaret. También se incorpora como socia la agente aduanal en Cancún y actual propietaria del hotel de lujo Casa de los Sueños, en Isla Mujeres, 
Alma Flores Castrejón, quien funge como directora de imagen del grupo hasta 2006, cuando cede su paquete accionarial (2\%) a Eduardo Albor Villanueva, socio desde 1999 de Dolphin Discovery, empresa fundada en 1994 por Lewis Brewer y Mike Wood, dedicada a la gestión de delfinarios (Flores, 2011).

En diciembre de 1992, tras casi dos años de obras, el parque Xcaret es inaugurado. En 1994, Quintana Pali, asociado con los hermanos Constandse Madrazo y con Francisco Córdova Lira, cuñado de uno de ellos, obtiene la concesión para desarrollar el parque ecológico de Xel-Há, un temprano intento de reproducir el ya exitoso modelo de Xcaret.

En 1994 crea la asociación civil Flora, Fauna y Cultura de México, que encabeza su hermana Guadalupe. Se trata de una organización no gubernamental (ONG) bajo el resguardo de Grupo Xcaret dedicada a la concientización y promoción de los valores medioambientales a través de diversas acciones, entre ellas el Programa de Protección y Conservación de Tortugas Marinas de la Riviera Maya, que desde 2002 realiza cada año el Festival de la Tortuga Marina y toda una serie de actividades de gestión comunitaria, como la creación de un centro formativo en Chemuyil, con el patrocinio de Microsoft; el parque La Ceiba, en Playa del Carmen, o la reforestación de manglares en la laguna de Nichupté, en Cancún.

Un análisis detallado de esta ONG revela que cumple algunas funciones que forman parte del concepto integral de gestión turística creado por Quintana Pali. Por un lado, constituye un elemento más en la estrategia de relaciones públicas de esta empresa que trata de integrar los conceptos de desarrollo sostenible y responsabilidad social en su funcionamiento diario (Molleda y Moreno, 2004; Moreno, 2006). La colaboración tan estrecha con una ONG parece la forma más diáfana de apalancar esos conceptos. Por otro lado, lleva a cabo acciones de difusión científica de lo que se realiza en los parques temáticos, e incluso mantiene actividades de seguimiento y control de un programa de investigación desarrollado por una entidad pública, el Centro de Investigaciones de Quintana Roo (CIQROO), que, una vez desmantelado, le fue traspasado a Grupo Xcaret en 1993 (Arenas et al., 1997; Arenas, Raigoza y Sánchez, 1998).

En 1997, Quintana Pali, asociado con su hermano Carlos, funda La Voz de México. Se trata de un paso más en la consolidación de un modelo de negocio turístico que ahora controla un elemento clave: la comunicación. Desde esa empresa se edita en español el periódico The Miami Herald, y se hace la revista Vive México, dirigida 
por su hermana Guadalupe y destinada al público turístico que arriba a Cancún y a la Riviera Maya. En 2008 contaba con un tiraje de 240000 ejemplares anuales, distribuidos en 7000 habitaciones de hotel. En ese mismo ámbito desarrolla Pixelpress, una compañía ubicada en Cancún dedicada a la impresión y producción de folletería y revistas para el sector turístico. Para ello, en 2001 se asocia con los hermanos Carlos y Alfredo Medina, dueños de la imprenta cancunense Tecnographics desde 1992. Actualmente, Pixelpress produce para Grupo Xcaret, los parques de Xel-Há, Grupo Dolphin Discovery y algunas cadenas hoteleras de Cancún.

En 2000 compra junto con un grupo de accionistas el rotativo La Crónica de Cancún, y le cambia el nombre por La Voz del Caribe. Esta adquisición fue objeto de controversia puesto que los propietarios eran el grupo hotelero Oasis, su socio Marcos Constandse Madrazo y el estado de Quintana Roo, gobernado en esos momentos por el controvertido Mario Villanueva Madrid, hoy encarcelado por sus vínculos con el narcotráfico. El diario se convirtió en instrumento de difusión política y, tras el cambio de gobernador, en instrumento empresarial de Quintana Pali, enfrascado en esos momentos en una ardua polémica derivada de su voluntad de construir un muelle de cruceros en Xcaret que no llegó a concretarse (Próspero, 2008). Este tabloide cerró sus rotativas en 2005 - luego del paso del huracán Wilma- por falta de inversión y el desinterés de sus propietarios por mantenerlo (Callejo Anzures, 2002; Guerrero, 2005).

En 1999, en una vuelta de tuerca más para el control del negocio turístico Xcaret, crea Cultura y Tradiciones de México, firma dedicada a la gestión y programación del Teatro de Cancún, del que es propietario. Esa misma empresa administró desde septiembre de 2001 el museo-tienda La Casa del Arte Popular Mexicano, manejado por su hermana Guadalupe, con una colección de 3400 piezas de artesanías de todo México, compradas ex profeso, que se expusieron hasta 2008 en un local de 500 metros cuadrados en la zona del embarcadero, en el Boulevard Kukulcán, cerca de la zona hotelera de Cancún. Esta iniciativa debe ser entendida más como una promoción y venta in situ de artesanías que como un centro museístico, a pesar de tener un programa de visitas escolares. Según los administradores, de 2001 a 2007 habían asistido más de 12000 niños. La exhibición está hoy instalada en la hacienda henequenera construida en Xcaret a manera de museo. Asimismo, Cultura y Tradiciones de México actúa como proveedora de artesanías en las tiendas de los parques de ocio del grupo (Ochoa, 2004). 
Entre 2006 y 2008 Grupo Xcaret reestructura su accionariado con la incorporación de Eduardo Albor Villanueva, director general de Dolphin Discovery, quien sustituye a Alma Flores Castrejón, la cual se integra a Grupo Vía Delphi. Un movimiento similar hará Francisco Córdova Lira al canjear sus acciones de Promotora Xcaret por otras de Grupo Vía Delphi, del que es director general.

\section{Algunos comentarios sobre la organización en Xcaret Respecto al modelo de negocio}

Detrás de Xcaret subyace un modelo empresarial sustentado en varias empresas destinadas a proporcionar suministros y servicios al parque (véase figura 1). Destaca sobremanera el papel de la comunicación y la mercadotecnia, elementos clave en la estrategia de ventas del parque y el posicionamiento de la marca. La gestión

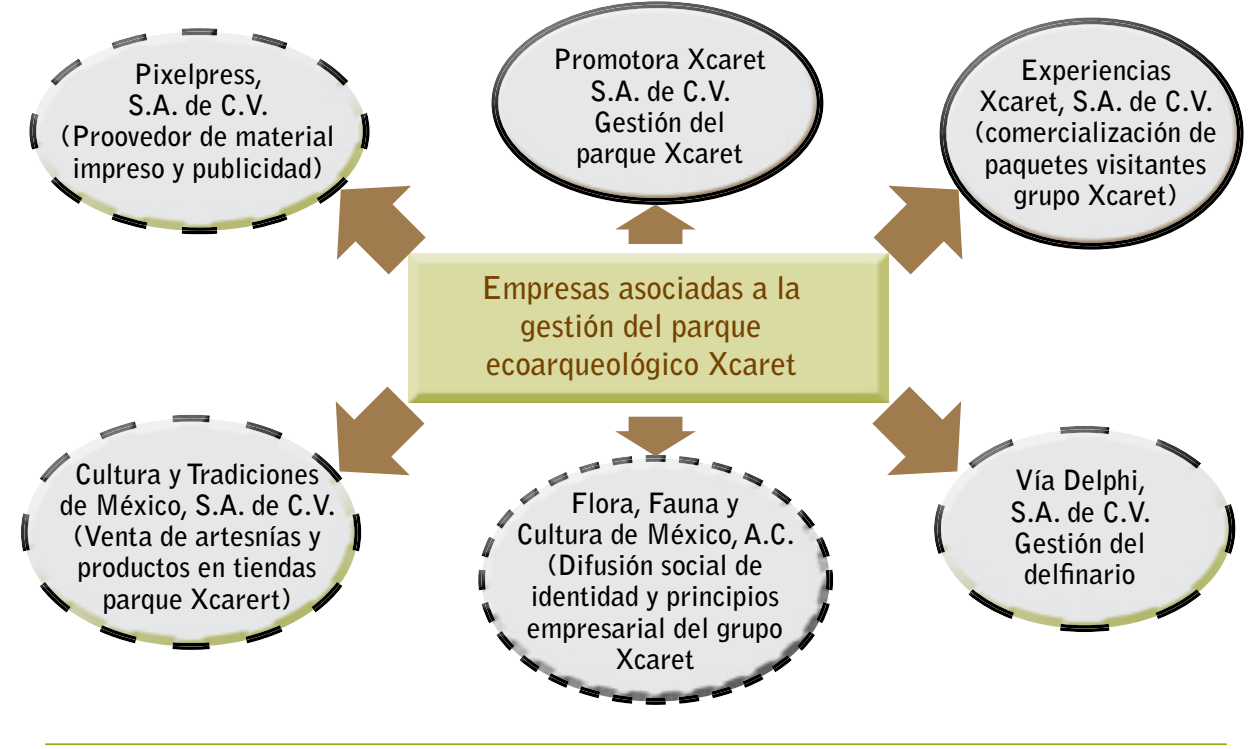

FIGURA 1. Red de empresas asociadas a la gestión del parque ecoarqueológico Xcaret

Nota: Con líneas discontinuas señalamos las empresas que suministran servicios al parque pero no forman parte de su estructura accionarial.

Fuente: Elaboración propia. 
de todas las facetas del parque es efectuada por Promotora Xcaret, mientras que la comercialización de los paquetes de visitantes, no sólo del parque sino de todo el grupo, por Experiencias Xcaret. En tanto, Vía Delphi, Pixelpress y Cultura y Tradiciones de México, relacionadas con el grupo por la presencia de alguno de sus accionistas, fungen como proveedoras de bienes y servicios necesarios para el funcionamiento del parque. Cabe mencionar a la asociación civil Flora, Fauna y Cultura de México en cuanto difusora de los planteamientos éticos de Grupo Xcaret.

\section{Respecto a una red empresarial asociada}

En primer término, conviene decir que detrás del desarrollo de Xcaret se encuentra un grupo empresarial centrado en las figuras de Quintana Pali y los hermanos Constandse Madrazo, que de forma descentralizada gestiona otros establecimientos de similares características, como el parque ecoturístico Xel-Há, también en Riviera Maya, y el parque Xplor, inaugurado en agosto de 2009 con más de 50 hectáreas, situado a dos kilómetros de Xcaret y dotado de un entorno de cavernas y cenotes al servicio de la explotación turística. Hasta hace algunos años formaban parte del grupo el parque El Garrafón, en Isla Mujeres, vendido en 2006 a Grupo Dolphin Discovery, y el parque del Cañón del Sumidero, en Chiapas, localizado en el municipio de Usumacinta, creado en 2001 con la participación como socio técnico de Grupo Ritco de Ecodesarrollo, firma de Grupo Alquimia, y con las aportaciones de los siguientes socios: Fondo Chiapas, fórmula de captación de inversiones del estado de Chiapas y de otros inversores privados; Corporación Financiera Internacional (CFI); Grupo Empresarial El Porvenir (GEP) y Grupo Xcaret. De igual manera, el grupo ha incursionado recientemente en el segmento de los paquetes turísticos estructurados, ofreciendo visitas a las zonas arqueológicas de Chichén Itzá y Ek Balam, en Yucatán, o combinando visitas a los recintos de Tulum y Cobá con la entrada a sus parques de ocio.

En segundo término cabe mencionar que Grupo Xcaret sostiene vínculos accionariales con otras empresas turísticas, como Grupo Vía Delphi, gestor de cinco delfinarios en distintos lugares de la costa del Caribe mexicano y; Grupo Alltournative, dedicado al turismo de aventura en la zona de la Riviera Maya y que en 2007 abrió el complejo de Río Escondido, cercano a Xcaret, donde se hacen visitas guiadas por un río subterráneo; Grupo Ritco, que efectúa las obras 
y ampliaciones derivadas de las continuas inversiones para las mejoras que se hacen tanto en Xcaret como en los otros parques; Grupo Dolphin Discovery, con delfinarios en Cozumel, Isla Mujeres y Puerto Aventuras, en el Caribe mexicano; en el Pacífico, en Nuevo Vallarta (Sealife Park Vallarta); en las Islas Vírgenes Británicas, Tórtola, y Anguila, y hasta 2007, el Sealife Park Hawaii, en Oahu, Hawai, vendido a la empresa Parques Reunidos. Esta última filiación parece tener un carácter mucho menos estructurado que las otras, no obstante, la red empresarial asociada muestra cómo el negocio de los servicios turísticos de ocio en el norte de Quintana Roo está en manos de unos cuantos empresarios, que en la mayoría de los casos mantienen vínculos societarios entre ellos.

\section{Respecto al organigrama}

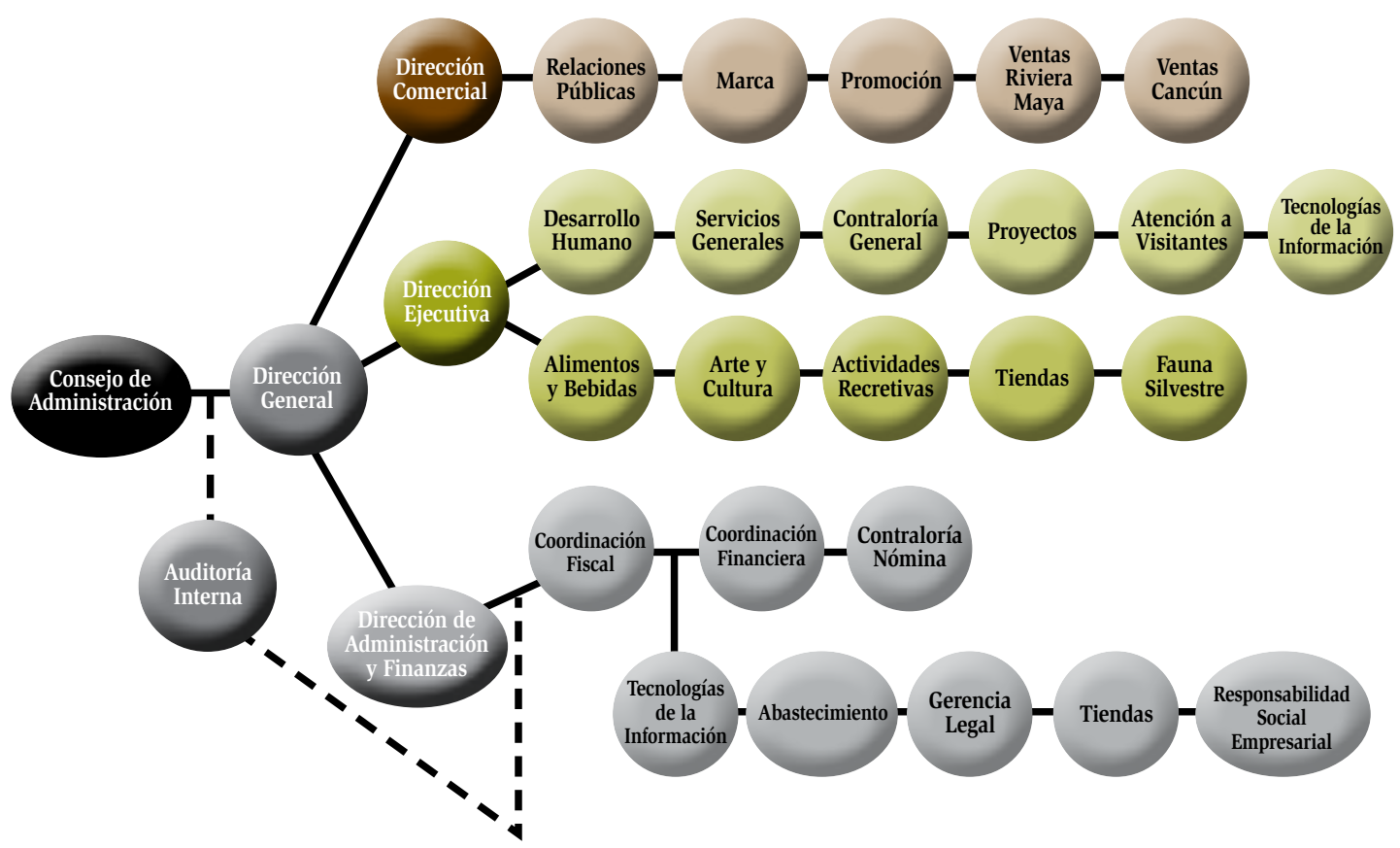

Figura 2. Organigrama de Promotora Xcaret (Grupo Xcaret) 
El organigrama que sustenta el manejo del parque ecoarqueológico Xcaret evidencia el amplio sentido jerárquico de esta organización. Tres elementos se sitúan en la cúspide y dirigen los destinos de la firma. En la parte más elevada, encontramos un Consejo de Administración conformado por cinco socios, tres de los cuales tienen vínculos familiares. De ese consejo pende una Dirección General desde la que se articulan tres direcciones específicas: Comercial, Ejecutiva y de Administración y Finanzas. Entre el Consejo de Administración y la Dirección General se ubica la figura de una Auditoría Interna, orientada a la fiscalización y control de la actividad financiera de la empresa.

Desde esas direcciones se articulan todos los departamentos dedicados a las actividades específicas de la firma, como puede verse en la figura 2. Respecto a esos departamentos, llama la atención la voluntad de segmentación de las actividades que se trasluce al analizar el organigrama. Ésta coincide con la búsqueda de nichos de mercados que realiza el grupo en sus estrategias de captación de clientela. Del mismo modo, destaca la gestión comercial regionalizada en dos espacios geográficos concretos (Cancún y Riviera Maya), dado que de éstos surge el mayor número de visitantes al parque. Por un lado, se deja intuir en ello la vinculación de turoperadores, hoteles y diversos agentes del sector turístico de Quintana Roo con los gerentes de ventas del parque, como ya señalábamos en líneas precedentes, y por otro, se vislumbra una operación comercial que camina entre lo pactado (el paquete de visita es concertado en destino) y lo coyuntural (se ofrece la visita en el momento en que el turista o el grupo se encuentra en algún lugar del estado).

Asimismo, el departamento de relaciones públicas pone énfasis en la creación, desarrollo y mantenimiento de toda una serie de estrategias que busca colocar la empresa entre ciertos agentes político-sociales que sirven para desarrollar las estrategias de crecimiento del grupo en sus distintos parques.

No sorprende tanto, atendiendo a las características del parque, la existencia de áreas dedicadas a la gestión y manejo de la fauna que se localiza en él (delfines, tortugas, guacamayas, etc.), a la gestión y organización de los espectáculos de sesgo cultural e historicista, o al mantenimiento de espacios que recrean un determinado ambiente histórico.

Un aspecto más, si observamos el organigrama, merece ser señalado: hay un departamento dedicado al desarrollo humano y una jefatura de responsabilidad social corporativa. Respecto al primero, baste decir que Grupo Xcaret mantiene una 
política de formación de gran parte de su personal, cuando menos, reseñable. Para los ejercicios de 2007 y 2008, la empresa reporta horas de formación específica para sus empleados (véase cuadro 2). En 2008, Xcaret contaba con 1665 trabajadores directos, con un índice de rotación de 25.5 \% (Grupo Xcaret, 2008). Algunos de ellos pueden considerarse especializados o técnicos puesto que interactúan en espacios como el delfinario, el mariposario, los sistemas de gestión de agua del parque, etc. En cuanto a la formación, se trata de programas intensivos de entrenamiento en diferentes niveles, desde educación básica hasta capacitación ejecutiva, seminarios de motivación y talleres de calidad en el servicio, seguridad en el trabajo, cultura y otros temas. La empresa mantiene además un equipo que proporciona educación gratuita - tanto materiales como tutores necesarios- para que todos los empleados tengan como mínimo la educación secundaria. Los idiomas también son parte de la capacitación. Asimismo, se ofrecen charlas informativas sobre cuestiones de salud, enmarcadas en un plan de salud integral para todo el personal (Santana Medina, 2010: 27).

\section{CUADRo 2. Distribución empleado / capacitación Promotora Xcaret (Grupo Xcaret)}

\begin{tabular}{lrr} 
& 2007 & 2008 \\
Horas curso capacitación & 126559 & 169860 \\
Horas curso por colaborador & 84 & 102 \\
Número de empleados & 1506 & 1665 \\
\hline
\end{tabular}

Fuente: Grupo Xcaret, 2009: 10.

Hay que añadir que Grupo Xcaret tiene firmados convenios de colaboración tanto con el Colegio Nacional de Educación Profesional Técnica (Conalep) en Quintana Roo y Yucatán (La Revista Peninsular, 2011), como con el Colegio de Estudios Científicos y Tecnológicos (Cecyte) de Quintana Roo, mediante los cuales los estudiantes de esos centros pueden llevar a cabo su servicio social y sus prácticas profesionales en los parques del grupo. También los tiene para prácticas profesionales de estudiantes con el Instituto Tecnológico Superior de los Ríos, en Balancán, Tabasco; con dos universidades privadas vinculadas al Consorcio Educativo Anáhuac: la Universidad Anáhuac, campus Cancún, y las sedes estatales de la Universidad Interamericana para el Desarrollo (UNID), y con la universidad 
pública estatal Universidad del Caribe, en Cancún, de cuya Junta de Gobierno es miembro uno de los principales socios de Grupo Xcaret.

En torno a la responsabilidad social, de 2004 a la fecha el grupo acredita el distintivo empresa socialmente responsable (ESR), el cual otorga el Centro Mexicano para la Filantropía (Cemefi), y que responde a la consideración por parte de la empresa de 120 parámetros centrados en aspectos como ética empresarial —entendida como el buen trato con proveedores y accionistas-, cuidado y preservación del medio ambiente, calidad de vida en la empresa, y vinculación con la comunidad (Porto y Castromán, 2006). En México, se trata de uno de los escasos ejemplos de firma turística que lo posee y que no es filial de trasnacionales o de compañías de gran tamaño (Saldaña Rosas, 2009: 8). En este contexto, desde 2007 hasta hoy Grupo Xcaret ha implementado el Modelo de Equidad de Género del Instituto Nacional de las Mujeres, un exhaustivo proceso de auditorías que certificaron el compromiso del grupo para que sus colaboradores tengan las mismas oportunidades de crecimiento independientemente de su edad y género. Además, el modelo justifica toda una serie de acciones concretas en favor del personal, como permisos de paternidad y por enfermedad o para atender los asuntos escolares de los hijos, etc. (Abreu, 2009: 167).

Asimismo, la empresa indica en sus balances diversas acciones con la comunidad de su entorno - ya sea directamente o a través de la ONG Flora, Fauna y Cultura de México, que podemos considerar una firma más del grupo- relacionadas con la protección del medio ambiente y focalizadas en la construcción de un parque urbano, La Ceiba, en Playa del Carmen, y en un programa de protección y seguimiento de tortugas marinas. También la empresa ha firmado el United Nations Global Compact, un programa de las Naciones Unidas para aplicar determinados valores éticos en el manejo empresarial.

Dadas las paradojas y ambivalencias que la responsabilidad social empresarial aún presenta en México, debe hacerse un mínimo análisis de las acciones que Grupo Xcaret desarrolla en ese rubro (Saldaña Rosas, 2009: 5). La lectura de los balances más recientes del grupo, los de 2007 y 2008, propone una imagen volcada a la defensa de ciertos valores éticos: cuidado del personal, vinculación con el entorno, atención y defensa del medio ambiente. Este último aspecto parece ser el que más incidencia tiene dentro del grupo, aquí relacionado con el concepto de sustentabilidad en todos los procesos operacionales de la empresa. Baste decir 
que la misión como empresa ("Ser únicos en recreación turística sustentable”) deja bien claro el concepto ideológico que modula la operación en los parques del grupo y en concreto en Xcaret (Khafash y Fraga, 2011: 150 y ss.).

Por un lado, estamos ante una respuesta empresarial coherente. La conservación del entorno donde se ubica el parque en las condiciones más adecuadas para su continuidad en el futuro garantiza el recurso de generación económica y la vida de la empresa (Abreu, 2009: 165-167). Por otro, se busca un posicionamiento, un tanto justificador, del uso excesivo del entorno. El concepto de la sustentabilidad en los procesos y los parámetros de responsabilidad social busca también la captación de clientes por medio de una imagen determinada y específica; facilita y justifica las nuevas inversiones en este parque y en los otros que gestiona el grupo, y afianza una imagen de coherencia entre la generación de beneficios y la responsabilidad con el medio donde opera. Todo ello convierte a Xcaret en referencia entre los poderes públicos - más aún en el caso de Quintana Roo-, siempre proclives a establecer niveles de afiliación con los empresarios más prominentes. En definitiva, el desarrollo de la responsabilidad social en Grupo Xcaret busca una relación de ganancia óptima con algunos actores que forman parte de su negocio: clientes, turistas, proveedores, poderes públicos y, en menor medida, con sus propios trabajadores y con la comunidad. Sin embargo, no parece que el desarrollo del concepto sea completo a cabalidad según los parámetros impuestos por el Cemefi, aspecto que coincide con muchas de las empresas que han obtenido la distinción ESR en otros lugares del país (Barroso Tanoira, 2008: 90).

Como veíamos, otro punto importante en la gestión de Grupo Xcaret es un marco ideológico que tiene la voluntad de promover la sustentabilidad y el respeto al medio ambiente dentro de una empresa turística (Córdova Lira, 2005 y 2006). Así, en el organigrama de la empresa gestora del parque y el de las otras del grupo directamente implicadas en la administración de los parques Xel-Há y El Garrafón pueden encontrarse gerencias de desarrollo sustentable, e incluso se articuló, a partir de 2004, un comité que las coordina. Esto es una forma de apropiación del discurso ambientalista como justificación de las acciones de cambio del entorno natural con motivo de la construcción de los parques (Redclift, 2001: 125). También es una manera de proceder que deriva en la activación de capacidades para generar utilidades y conocimiento técnico propio. Tal es el caso de la existencia de un delfinario y la necesidad de mantener a los cetáceos, lo cual ha conducido 
al conocimiento y la cría de ejemplares en cautiverio (Castello, Alaniz y Vega, 2007). En esta cuestión, el grupo empresarial se ha convertido en pionero y referente en la cría de delfines por haber obtenido beneficios de ese conocimiento en el parque Xcaret o en otros del grupo. Esa experiencia, por ejemplo, puede ser confrontada en estos momentos con las empresas competidoras, gestoras de delfinarios y proveedoras de los animales, acusadas de turbios manejos y falta de ética en el cuidado de las especies (Flores, 2011).

Los planteamientos de sostenibilidad en las empresas del grupo derivan en una obligación de preservar la base ecológica de los parques fortaleciendo ciertos métodos de prestación de servicios como elemento para asegurar el futuro de la firma. A su vez, el grupo empresarial, mediante ciertas estrategias que lindan con la filantropía, las relaciones públicas y la comunicación, interviene de diversos modos en el entorno socioeconómico que lo rodea. Todo ello ha hecho aflorar el concepto de responsabilidad social empresarial como una señal de identidad de Grupo Xcaret. Una de sus estrategias es el mecenazgo de programas de conservación ambiental instigados por la asociación civil Flora, Fauna y Cultura de México, que, como hemos observado, se ha convertido en una herramienta de las estrategias de la empresa, especialmente en materia de difusión social de los valores emanados de la sustentabilidad y la responsabilidad social empresarial. Asimismo, vertebra, bajo la fórmula de convenio, distintos proyectos de investigación con centros y universidades del país que están asociados a elementos existentes en el parque y con otros que afectan la realidad regional. Éste sería el caso de los convenios firmados con el Instituto de Geofísica de la UNAM para el monitoreo de la calidad de los acuíferos kársticos de la zona norte de la Península de Yucatán; los trabajos sobre la deriva de las larvas del caracol rosa por parte del Instituto Politécnico Nacional y del Florida Marine Research Institute, y un programa de reproducción en cautiverio del flamenco rosa de la reserva natural de río Lagartos, endémico de ese lugar e introducido en Xcaret.

\section{Algunas conclusiones}

El parque ecoarqueológico Xcaret se nos revela como un ejemplo emblemático, fruto de la hibridación y la readaptación tanto de espacios naturales como de elementos patrimoniales en aras de una oferta turística que combine esos aspectos con 
actividades lúdicas relacionadas con el turismo de sol y playa, propias de la Riviera Maya. Pero la estrategia de Xcaret también debe considerar la captación de públicos en un entorno muy competitivo, hecho que requiere agresividad comercial y un claro posicionamiento de la empresa y sus marcas; en este caso, centrándose en los valores del desarrollo sustentable y la responsabilidad social empresarial. Además, la propia trama empresarial gestada por los fundadores del parque permite un control de los variados mecanismos de atracción de visitantes y la dotación continuada de inversiones para incrementar esa visibilidad. A eso se añade una red de relaciones entre los socios del grupo que determinan el control de diversos segmentos turísticos que operan en la Riviera Maya.

Finalmente, Xcaret mantiene una postura ambivalente en cuanto modelo de servicio turístico respetuoso con el medio ambiente y en su aplicación del desarrollo sustentable. Si consideramos toda su operativa se descubre como un caso de explotación más o menos controlada de dos de los mayores recursos disponibles en México: la diversidad natural y la riqueza cultural, a la vez que sigue los procedimientos y mecanismos propios del empresariado mexicano, la creación de redes como mecanismo de control del mercado, y el crecimiento sistemático de los rendimientos aprovechando la extensión y fortaleza de las redes creadas. 


\section{FUENTES CONSULTADAS}

Abreu, J. L. (2009). "Situación actual de la RSE en el sector turístico mexicano". Daena: International Journal of Good Conscience, 4 (2), septiembre de 2009, 160-173.

Aguilar, A. (1999). "Miguel Quintana, Xcaret y ocho años de continuo crecimiento". Diario Reforma, sección "Negocios”, 9 de marzo de 1999, 4.

Arenas, A., R. Raigoza y R. Sánchez (1998). "Comparison of Growth Curves for Sea Turtles of two nest in Captivity and Follow of Evolution of Living tag Technique in Green Turtles Chelonia Mydas at Xcaret Eco-Archeological Park", en F. Abreu et al. (comps.). Proceedings of the Eighteenth International Sea Turtle Symposium. 3-7 de marzo de 1998. Mazatlán: noaa, 159-161.

Arenas, A., et al. (1997). "The Sea Turtle Program of Xcaret, the Eco-Archeological Park in Quintana Roo, México", en S. Epperly y J. Braun (comps.). Proceedings of the Seventeenth Annual Sea Turtle Symposium. 4-8 de marzo de 1997. Orlando: National Oceanic and Atmospheric Administration (NOAA), 126-130.

Barroso Tanoira, F. G. (2008). "La responsabilidad social empresarial: Un estudio en cuarenta empresas de la ciudad de Mérida, Yucatán”. Contaduría y Administración, 226, 73-91.

Callejo Anzures, J. A. (2002). De Cancún a Almoloya: El imperio roto de Mario Villanueva. México: Océano.

Campos Cámara, B. L. (2007). Proceso de urbanización y turismo en Playa del Carmen, Quintana Roo. Chetumal: Universidad de Quintana Roo (uqroo)/Plaza y Valdés.

Castello, H., Y. Alaniz y C. Vega (2007). Los delfinarios en México. Un informe crítico [en línea]. Disponible en: http://burica.files.wordpress.com/2007/03/ delfinarios-en-mexico.pdf

Checa-Artasu, M. (2008). "La gestión del patrimonio cultural reinventado: los casos del parque ecoarqueológico de Xcaret (Quintana Roo, México) y la ciudadela ibérica de Calafell (Tarragona, España)”. Ketzalcalli, 1. Quintana Roo: Departamento de Ciencias Sociales-uqroo/University of Hamburgo/ Institute of Mesoamericanistics, 113-127. 
- (2009). "Patrimonio, naturaleza recreada y gestión turística: el parque ecoarqueológico de Xcaret (Quintana Roo)”. ARA. Journal of Tourism Research/Revista de Investigación en Turismo, 2 (1), 45-58. Barcelona: Fundación Global Democracia y Desarrollo/Universitat de Barcelona.

Córdova Lira, F. (2005). "Aprovechando el potencial de los mercados verdes. Industria turística”. Mexico Investor Forum, octubre de 2005. (2006). "Educación para el desarrollo". Mesa redonda en el IV Simposio de Responsabilidad Social en las Américas, julio de 2006.

Córdova Ordoñez, J. y A. García Fuentes (2003). “Turismo, globalización y medio ambiente en el Caribe Mexicano”. Investigaciones Geográficas, 52, diciembre de 2003, 117-136.

Cortés de Brasdefer, F. (2005). "El parque ecoarqueológico de Chakanbakán, Quintana Roo, México”, en D. Magaloni Kerpel (comp.). Lineamientos y limitaciones en la conservación. Pasado y futuro del patrimonio. México: Universidad Nacional Autónoma de México, 255-284.

Flores, V. (2011). “Dolphin Discovery, imperio de corrupción”. Diario Respuesta, 9 de febrero de 2011, 10-11.

Grupo Xcaret (2008). Amar a México. Balance Social, Ambiental y Cultural 2007 [en línea]. Cancún: Jefatura de Responsabilidad Social Empresarial. Disponible en: http://www.rodolfosagahon.com/docs/balancexc.pdf (2009). Balance Social, Ambiental y Cultural 2008 [en línea]. Cancún: Jefatura de Responsabilidad Social Empresarial. Disponible en: http://www. rodolfosagahon.com/docs/librobalance2008.pdf

Guerrero, S. (2005). "Los negocios de Miguel Quintana Pali con el ex gobernador Mario Villanueva”. Expresión Libre, $1^{\circ}$ de junio de 2005, 2.

Howard, G. (2002). "Buscador y diseñador de ideas". El Universal, 21 de enero de 2002.

INEGI (2005). Conteo de Población y Vivienda 2005. Aguascalientes: Instituto Nacional de Estadística y Geografía.

Khafash, L. y J. Fraga (2011). "Entornos naturales y mercado ecoturístico en la Riviera Maya: Xcaret y Xel-Há en Quintana Roo, México”, en L. Prats y A. Santana (coords.). Turismo y patrimonio, entramados narrativos. Tenerife: Asociación Canaria de Antropología/Pasos, Revista de Turismo y Patrimonio Cultural, 145-165. 
La Revista Peninsular (2011). "Grupo Xcaret y Conalep firman convenio en beneficio de los futuros profesionales". La Revista Peninsular, 1110, 4 de febrero de 2011 [en línea]. Disponible en: http://www.larevista.com.mx/ediciones/1110/grupo-xcaret-conalep-firman-convenio-beneficio-los-futuros-profesionales-23134/ [2011, 10 de noviembre].

Lira, R. (2002). "Miguel Quintana Pali, inventor de paraísos”. Casas \& Gente, 163, 7. Martí, F. (1985). Cancún: Fantasía de banqueros. México: Editora Uno.

Mateos Vega, M. (2007). "INAH: Xcaret propone una versión hollywoodiense de la cultura maya”. La Jornada, 26 de enero de 2007, 28.

Meraz, F. (2008). "Promotora Xcaret pagará impuestos tras 20 años”. Milenio Diario, 16 de noviembre de 2008, 16.

Meza, M. A. (2010a). "Marcos Constandse: la ética del bien en la vida y en los negocios”. Liderazgo. Alto Nivel en los Negocios en Quintana Roo, 24 de marzo de 2010 [en línea]. Disponible en: http://www.liderazgoonline.mx/marcosconstandse-etica-bien-vida-negocios [2011, 13 de noviembre].

(2010b). “Grupo Ritco de Eco-Desarrollo, S. A. de C. V.”. Liderazgo. Alto Nivel en los Negocios en Quintana Roo, 23 de marzo de 2010 [en línea]. Disponible en: http://www.liderazgoonline.mx/grupo-ritco-eco-desarrollo-sa-c-v [2011, 13 de noviembre].

Molleda, J. C. y A. Moreno (2004). "Relaciones públicas y desarrollo sostenible. Estudio de caso del grupo Xcaret en México”. Ponencia presentada en el I Congreso Anual de Investigación en Relaciones Públicas, 17-18 de noviembre. Sevilla.

Moreno, A. (2006). "Relaciones públicas y desarrollo sostenible en el sector turístico. El caso de Xcaret en el Caribe Mexicano”, en M. T. Otero Alvarado. Investigación y relaciones públicas. Madrid: Asociación de Investigadores en Relaciones Públicas/Universidad Complutense de Madrid, 76-93.

Ochoa, S. (2004). "De tal palo tal astilla”. Latitud 21. Líderes y Negocios en el Caribe Mexicano, 2 (15), junio de 2004 [en línea]. Disponible en: http://www. latitud21.com.mx/junio04/galeria.html [2011, 10 de noviembre].

Porto, N. y J. Castromán (2006). "Responsabilidad social: un análisis de la situación actual en México y España”. Contaduría y Administración, 220, septiembre-diciembre de 2006, 67-87. 
Próspero, V. (2008). "Compás de espera”. Latitud 21. Líderes y Negocios en el Caribe Mexicano, 6 (67), octubre de 2008 [en línea]. Disponible en: http:// www.latitud21.com.mx/noviembre08/reportaje_especial.html [2011, 8 de noviembre].

Ravelo, A. y D. Estolano (2004). Estudio de Mercado Permanente para el Estado de Quintana Roo como Destino Turístico. Marzo 2003-Febrero 2004. Cancún: Instituto de Investigaciones Turísticas-Universidad La Salle Cancún.

Razo Ruiz, A. L. (2004). La administración del patrimonio cultural. El caso de Xcaret, Quintana Roo. Tesis de Maestría en Ciencias Sociales Aplicadas a los Estudios Regionales. Chetumal: UQROO.

Redclift, M. (2001). “'Changing Nature': The Consumption of Space and the Construction of Nature of the 'Mayan Riviera'”, en M. Cohen y J. Murphy (eds.). Exploring Sustainable Consumption. Environmental Policy and the Social Sciences. Oxford: Emerald Group Publishing, 121-133.

Rodríguez, P. (2000). Xcaret. Cancún: Promotora Xcaret.

Saldaña Rosas, A. (2009). "La empresa socialmente responsable en México: auge, paradojas y perspectivas”. Ciencia Administrativa, 2, 1-8. Veracruz: Instituto de Investigaciones y Estudios Superiores de las Ciencias Administrativas-Universidad Veracruzana.

Sánchez, M., E. Jordán y R. Raigoza (2000). “Development of Created Coral Reef Habitats at Xcaret Aquarium”. Bulletin de l'Institut Océanographique, 20 (2), 187-192.

Santana Medina, J. L. (2010). La responsabilidad social empresarial en el turismo: una aproximación desde la nueva teoría institucional. Tesis de Doctorado en Ciudad, Territorio y Sustentabilidad. Guadalajara: Centro Universitario de Arte, Arquitectura y Diseño-Universidad de Guadalajara.

Wyllys Andrews, E. y A. P. Andrews (1975). A Preliminary Study of Ruins of Xcaret, Quintana Roo. Nueva Orleans: Middle American Research Institute-Tulane University. 